\title{
Numerical Study on Control of Tollmien-Schlichting Waves Using Plasma Actuators
}

\author{
Marios Kotsonis* Rogier Giepman ${ }^{\dagger}$ and Leo Veldhuis ${ }^{\ddagger}$ \\ Delft University of Technology, Delft, 2629HS, The Netherlands
}

\begin{abstract}
A numerical investigation on the use of plasma actuators for transition control is presented. The numerical framework involves the solution of the full unsteady $2 \mathrm{D}$ incompressible Navier Stokes equations using a finite volume formulation. The set of equations is formulated by solving for the perturbations in the flow while a mean laminar boundary layer flow is considered fixed and superimposed. The effect of the plasma actuator is represented as an imposed unsteady body force distribution derived from experimental measurements. Furthermore, an adaptive control system based on the filtered-x LMS algorithm is implemented directly into the flow solver. The control system uses pressure signals at the wall in order to compute the frequency, phase and amplitude of the plasma body force which minimizes the intensity of the propagating TS waves. Results show large reductions in wave amplitude for both single and multi frequency cases.
\end{abstract}

\section{Introduction}

Plasma actuators have been studied extensively in recent years, as flow control devises. Their low power consumption, lack of moving parts and robustness render them ideal for flow manipulation. There are several implementations of the actuators the most popular being the Dielectric Barrier Discharge (DBD) kind. It is commonly accepted that some sort of collisional processes between the heavy plasma species (mostly ions) and neutral air is responsible for the momentum transfer. In a macroscopic scale, which is usually the scale of the flow to be controlled, the model of an exerted body force on the fluid seems to describe the effect reasonably.

Plasma actuators have been used in several studies aiming at separation control, ${ }^{1}$ turbulent drag reduction, ${ }^{2}$ boundary layer control ${ }^{3-5}$ and transition delay. ${ }^{6}$ Excellent reviews on plasma actuators for aerodynamic flow control have been published recently. ${ }^{7,8}$

The capabilities of the actuators suggest the feasibility of their implementation in several flow control scenarios. It is therefore desirable to have an efficient and accurate model of the effect of the actuator on a given flow. A large amount of simulation studies has been conducted in order to simulate and capture the underlying physics of the ionization process. ${ }^{9,10}$ These vary in model complexity, from simple phenomenological models to first principles fluid models. ${ }^{11}$ Extended simulations for multi-species fluids have also been investigated. ${ }^{12}$ In the majority of these modeling approaches the final goal is to determine the exerted body force on the fluid. A number of studies on flow solvers implementing plasma actuators have used this approach successfully to couple the effect of the actuator with the flow dynamics. ${ }^{?, 13}$ More recently, the authors, ${ }^{14}$ have developed a technique which enables the measurement of the two-dimensional body force field experimentally for a selected applied voltages and carrier frequencies.

One of the promising concepts for the utilization of plasma actuators is the cancellation of TollmienSchlichting waves in a transitional boundary layer. This technique aims at tackling the instability waves while still in linear amplification stage. At this stage the waves have little energy content with typical amplitudes of $0.01 \%$ of the freestream velocity. ${ }^{15}$ This technique has been investigated experimentally ${ }^{16,17}$ as well as numerically. ${ }^{18,19}$ In recent studies ${ }^{6}$ artificially introduced TS waves were successfully canceled using plasma actuators.

${ }^{*} \mathrm{PhD}$ researcher, Faculty of Aerospace Engineering, Kluyverweg 1.

$\dagger$ MSc candidate, Faculty of Aerospace Engineering, Kluyverweg 1.

${ }_{\ddagger}^{\ddagger}$ Associate Professor, Faculty of Aerospace Engineering, Kluyverweg 1, AIAA Member. 
In this paper a numerical investigation on the TS wave cancellation concept is performed. More specifically the actuator is used as a counter-wave generator operating in a transitional boundary layer. In the $2 \mathrm{D}$ case the instabilities propagating in the laminar boundary layer correspond to the unsteady TollmienSchlichting modes. Through correctly phased unsteady actuation the wave amplitude can be reduced leading to a delay of transition and overall skin friction drag decrease. For the unsteady actuation the plasma actuator is used through the concept of a volume distributed body force. In this study both a generic force distribution as well as experimentally derived body forces are used. For the selection of the correct actuation signal a stand-alone automatic control system employing the filtered-x LMS algorithm ${ }^{20}$ is implemented in the flow solver. The control system runs in parallel to the flow solver and the actuation is done in real time.

\section{Methodology}

\section{A. Numerical flow solver}

In order to resolve all the spatial and temporal scales involved in the transition problem, a full Navier-Stokes solution is pursued. Furthermore a reduced approach is followed in which the base flow (laminar boundary layer) is considered fixed while the NS equations are solved for the TS perturbations. More specifically consider the 2D incompressible Navier Stokes equations in the presence of body forces:

$$
\frac{\partial \vec{U}}{\partial t}+\vec{U} \cdot \nabla \vec{U}-\nu \nabla^{2} \vec{U}=-\frac{\nabla P}{\rho}+\frac{\vec{F}}{\rho}
$$

In the context of hydrodynamic stability it is often convenient to express the flow as the sum of a base mean flow $\left(\vec{U}_{0}, P_{0}\right)$ and a fluctuating component $(\vec{u}, p)$. the mean flow which is steady in time can be calculated beforehand using either analytical solutions of the Falkner-Skan equations or numerical solutions of the NS equations. The velocity, pressure and force fields are expressed as:

$$
\begin{aligned}
\vec{U}(x, y, t) & =\vec{U}_{0}(x, y)+\vec{u}(x, y, t) \\
P(x, y, t) & =P_{0}(x, y)+p(x, y, t) \\
\vec{F}(x, y, t) & =\vec{f}(x, y, t)
\end{aligned}
$$

Substituting (2) into (1) and subtracting the mean values produces the expression for the disturbances:

$$
\frac{\partial \vec{u}}{\partial t}+(\vec{u} \cdot \nabla) \vec{u}+\left(\vec{U}_{0} \cdot \nabla\right) \vec{u}+(\vec{u} \cdot \nabla) \vec{U}_{0}-\nu \nabla^{2} \vec{u}=-\frac{\nabla p}{\rho}+\frac{\vec{f}}{\rho}
$$

In this study the set of governing equations is implemented and solved within the computational framework of the open source code OpenFOAM. This platform employs second order finite volume discretization in space and a variety of finite difference methods for temporal advancement. The laminar boundary layer base flow $\left(U_{0}\right)$ can be calculated using the boundary layer equations or even the Falkner-Skan solutions. Nevertheless in this study the incompressible viscous solver of OpenFOAM is used since the computational cost is minimum for a $2 \mathrm{D}$ study.

The boundary conditions for the stability problem are Dirichlet conditions of zero perturbation velocity at the inflow, plate and top boundary while a Neumann condition of zero gradient is imposed at the outflow. For pressure, Neumann conditions of zero gradient are imposed on all boundaries except the top where fixed zero pressure is imposed.

In order to investigate the boundary layer stability problem the disturbance in question must be introduced in the numerical domain. This can be done in a multitude of ways such as imposed fluctuating velocity at the inflow, unsteady suction and blowing at the wall or fluctuating distributed body forces. It is generally a good choice to introduce the disturbance upstream of the first neutral curve to allow any transient noise to damp out. In this study two techniques for introducing the disturbance are employed. Firstly a fluctuating velocity component is imposed at the inflow. The shape of the fluctuation corresponds to the eigenfunction of the TS wave calculated from Linear Stability Theory for the given frequency. Zero net mass is achieved since the imposed velocity has zero mean value. This technique is useful for verification purposes as well as single frequency control cases. Nevertheless it presents some difficulties when wavetrains of more than one frequencies must be introduced. For these cases an unsteady horizontal body force $\left(f_{g}(x, y, t)\right)$ is used in the form of: 


$$
f_{g}(x, y, t)=\sum_{i}^{n}\left(W_{i} \cdot \sin \left(2 \pi \omega_{i} t\right)\right) \cdot \sin \left(\pi \frac{x}{x_{2}-x_{1}}\right) \cdot \sin \left(\pi \frac{y}{y_{2}-y_{1}}\right)
$$

where $x_{1}<x<x_{2}, y_{1}<y<y_{2}$.

The differences $\left(x_{2}-x_{1}\right)$ and $\left(y_{2}-y_{1}\right)$ define the spatial wavelengths of the force in $\mathrm{x}$ and $\mathrm{y}$ direction. For the current case the $x$ wavelength is approximately half the wavelength of the desired TS wave while the vertical wavelength is approximately a quarter of the TS wavelength. The temporal variation of the fluctuating force is controlled by the sum of $n$ sinusoidal modes with corresponding amplitudes $\left(W_{i}\right)$ and frequencies $\left(\omega_{i}\right)$. In general, the numerical study in this paper makes use of the imposed inflow for the single frequency cases while the imposed body force is used for all multi frequency cases.

\section{B. Automatic Control System}

For the control system the filtered- $x$ LMS (FXLMS ${ }^{20}$ adaptive algorithm based on FIR filters is applied. The concept is derived from active noise cancellation techniques and it involves a feed-forward loop for the estimation of the control signal which drives the actuator. More specifically, the system uses a reference and an error signal which in the investigated case are pressure values at the wall. A schematic of the working principle is given in Fig.1. The system is using a primary FIR filter $(\vec{w})$ through which the reference signal $(\vec{x})$ is passed as follows ${ }^{\mathrm{a}}$ :

$$
y(k)=\vec{w}(k)^{T} \cdot \vec{x}(k)=\sum_{i=0}^{L-1} w_{i}(k) x(k-i)
$$

where $k$ is the time instant of the last sample of the reference signal. $L$ is the length of the primary FIR filter $\vec{w}$. To be noted here that $\vec{x}(k)=[x(k) \quad x(k-1) \ldots x(k-L+1)]^{T}$ is a vector containing the $L$ last samples of the reference signal. Through this process the output signal $y(k)$ which is sent to the actuator is produced. In order to achieve the desirable cancellation effect the weights of the primary filter $\vec{w}$ must be adapted. This is achieved through a Least Mean Square (LMS) approach which compares a filtered version of the reference signal with the error signal at time instant $k$ in order to compute an updated set of primary filter coefficients for time instant $k+1$;

$$
\vec{w}(k+1)=\vec{w}(k)-2 \mu e(k) \vec{f}(k)
$$

where the convergence coefficient $\mu$ is a constant used to increase or decrease the convergence of the adaptation process. The choice of $\mu$ is extremely important for the final control performance. As will be shown too small $\mu$ leads to slow convergence and reduced performance while too large $\mu$ renders the algorithm unstable. For this study a variation of the standard FXLMS algorithm is used where $\mu$ is normalized by the strength of the reference signal in order to increase the robustness of the convergence process:

$$
\mu=\frac{\beta}{\vec{x}^{T}(k) \vec{x}(k)}
$$

where $\beta$ is a predifined constant.

In the control algorithm $\vec{f}(k)$ is produced by filtering the reference signal with a secondary FIR filter (FIRII) which is a digital representation of the natural cancellation path. This is done in order to have a valid comparison between reference and error signals for the update process. The cancellation path copy (FIRII) is obtained using a system identification routine based on a modified version of the LMS adaptive algorithm. More specifically the actuator is driven by a predetermined signal which in this case is the reference signal $x(k)$. At the same time $\vec{x}(k)$ is passed through the secondary filter. The produced signal is then subtracted from the error signal and used in the LMS routine to update the coefficients of FIRII. By using the difference of the two signal to update FIRII, the final weight coefficients of the secondary filter are such that $f(k)=e(k)$. This effectively means that FIRII can model the natural cancellation path as it was initially required by the primary control system. As soon as the system ID algorithm converges and FIRII is available the control sequence can begin.

\footnotetext{
${ }^{\mathrm{a}}$ For the description of the controller algorithm the notation of Hansen ${ }^{21}$ is followed throughout.
} 
Both the system identification and control algorithms are implemented in the OpenFOAM framework and operate in parallel to the flow solver. Several parameters of the algorithm such as primary and secondary filters lengths, sampling rate, weights update rate and convergence values $(\beta)$ are set prior to the simulation.

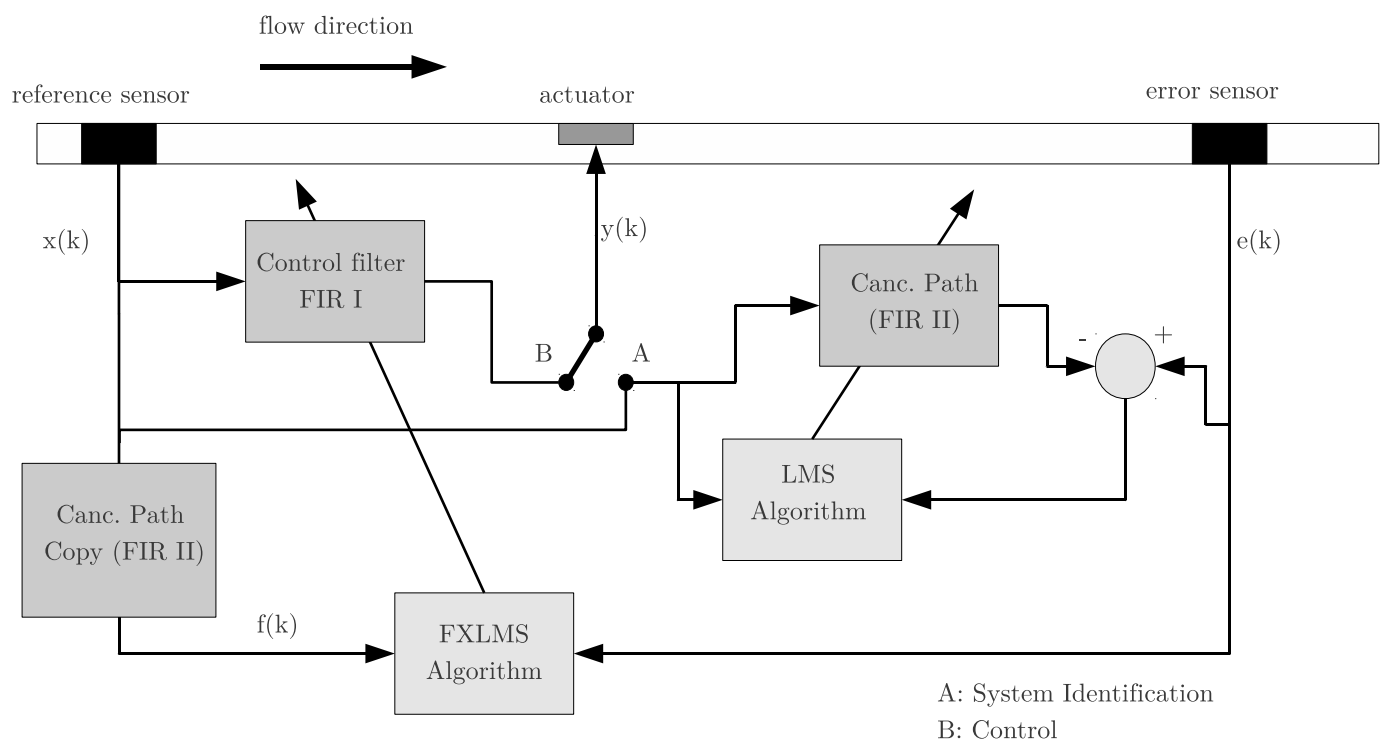

Figure 1. Schematic of the working principle for the filtered- $x$ LMS control algorithm.

\section{Body Force Implementation}

To effectively capture the effect of the plasma actuator a distributed volume body force is applied as described earlier. The magnitude and spatial distribution of the body force have been subject of numerical and experimental investigations. For this study two levels of approximations are applied to the force description. Firstly a generic force distribution with arbitrary amplitude and spatial extent is used in order to gain insight into the interaction between the three main components (stability, body force and controller) of the modeling process. The force shape is chosen as a half-sinus distribution in both $\mathrm{x}$ and $\mathrm{y}$ directions given by:

$$
f_{x}(x, y)=A_{x} \cdot \sin \left(\pi\left(\frac{x-x_{s}}{l}\right)\right) \cdot \sin \left(\pi\left(\frac{y-y_{s}}{h}\right)\right) \quad \text { for }: \quad x_{s} \leq x \leq x_{s}+l, \quad y_{s} \leq y \leq y_{s}+h
$$

where $A_{x}$ is the amplitude of the horizontal force component, $x_{s}$ and $y_{s}$ define the starting coordinates of the force distribution and $l$ and $h$ the length and height of the distribution respectively. The vertical force component $f_{y}$ is set to zero for simplicity. It should be noted here that the controller controls only the amplitude $\left(A_{x}\right)$ of the force and not the spatial extend. An example of the generic force distribution is given in Fig.2.

For the second level of approximation, the body force implementation is extended to incorporate experimentally derived force distributions from actual plasma actuators. ${ }^{14}$ In contrast

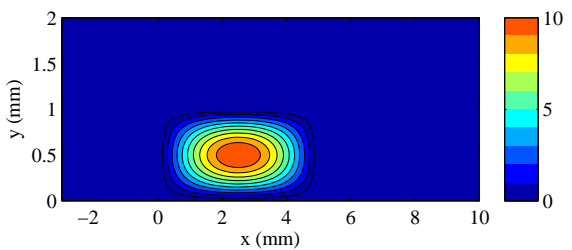

Figure 2. Example of a generic body force distribution with $l=4 \mathrm{~mm}, \mathrm{~h}=1 \mathrm{~mm}$ and $A_{x}=10 \mathrm{mN} / \mathrm{mm}^{2}$. to the generic force distribution, the plasma force distributions present additional complexities. More specifically, the control parameter here is not the amplitude of the force but the value of the applied voltage. This in turn defines not only the amplitude of the force distribution but also the spatial extent and the shape. The available experimental results cover a range of applied voltages from 8 to $16 k V_{p p}$ with steps of $2 k V$. In total 5 different force distributions are available. For implementation of these forces in the solver as a function of voltage, an interpolation based on 2D Bernstein 
surfaces is carried out and the generated coefficients are stored in the controller environment as lookup tables. This enables the on-line calculation of the body force for every value of applied voltage within the range of 8 to $16 k V_{p p}$. For values less than $8 k V_{p p}$ the body force is set to zero while for values larger than $16 k V_{p p}$ the force remains constant and equal to the $16 k V_{p p}$ case. A comparison between the original force distributions and the respective Bernstein surface models is presented in Fig.3.
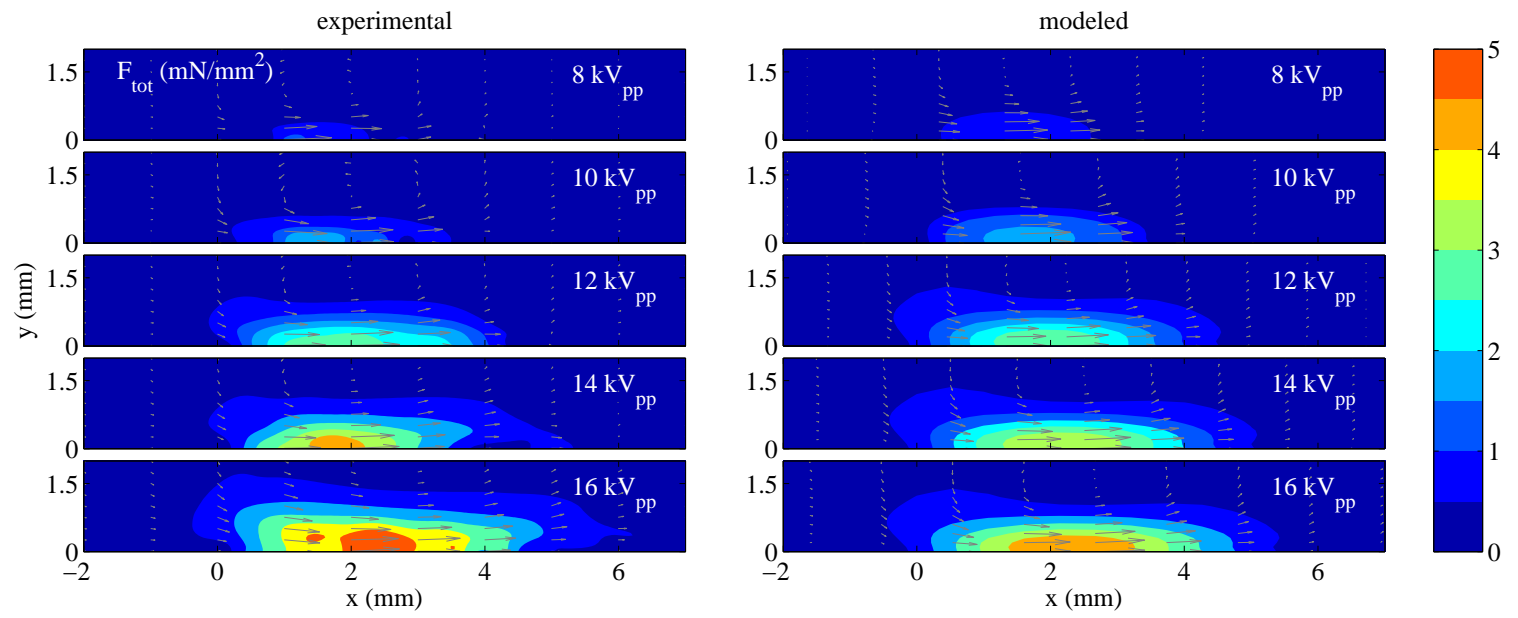

Figure 3. Experimental and respective modeled body force distributions for different applied voltages.

\section{Test cases and Mesh}

For the entire numerical study, flat plate boundary layers with zero pressure gradient at two freestream velocities are considered. These correspond to 10 and $30 \mathrm{~m} / \mathrm{s}$. This choice has been made for several reasons. Firstly, the $10 \mathrm{~m} / \mathrm{s}$ case (case A) is used to develop the controller system and gain insight on the influence of several parameters such as convergence constant and length of the body force on the final control performance. Furthermore, case A has been used for the verification of the numerical flow solver through various grid and boundary conditions studies. The body force used for the cancellation of the TS waves in case A is exclusively the generic sinusoidal distribution. Case B involves the implementation of the experimentally derived body force distributions in the transition control framework. As it is already mentioned, no control over the amplitude of this force exists other than the selection of the applied voltage. To this reason case B involves laminar flow at a larger Reynolds number in order to facilitate the increased force amplitude.

For all cases a Reynolds number based on the inflow displacement thickness is defined:

$$
R e_{0}^{*}=\frac{U_{\infty} \delta_{0}^{*}}{\nu}
$$

For case $\mathrm{A}, R e_{0}^{*}$ is 658 with an inflow displacement thickness $\delta_{0}^{*}=0.98 \mathrm{~mm}$. Three single frequency and one multi frequency stability and control sub cases are ran. A frequency number is set based on the Reynolds number at the inflow:

$$
F=\frac{\omega_{0}}{R e_{0}^{*}} \times 10^{6}
$$

where $\omega=\frac{2 \pi \delta^{*}}{U f_{r}}$ is the local non-dimensional frequency of the mode. The three single frequency sub cases correspond to $F=66,86$ and 106. Respective physical frequencies $\left(f_{r}\right)$ are 70, 91.2, and $112 \mathrm{~Hz}$ respectively. These modes have been chosen such that during introduction in the domain they are still upstream of the neutral curve and thus stable. A stability diagram for cases A and B, indicating the computational domain and the position of the studied modes, is shown in Fig.4.

Case B involves a higher inflow Reynolds number $\left(R e_{0}^{*}=1539\right)$. For this case one single and one multiple frequency sub case is ran. The single frequency case corresponds to mode $F=25$ while the multi frequency case involves the summation of modes $F=15,25$ and 35 with equal amplitudes during introduction. The respective physical frequencies for these modes are $f_{r}=143,238$, and $334 \mathrm{~Hz}$. 
Both cases are run on structured rectangular meshes. For both cases the total number of cells used is approximately 0.1 million. For case A the mesh extends from $x=0.2$ to $1.2 \mathrm{~m}$ and $y=0$ to $0.025 \mathrm{~m}$ while for case B the mesh extents from $x=0.4$ to $1.4 \mathrm{~m}$ and $y=0$ to $0.025 \mathrm{~m}$. The mesh is clustered linearly near the wall along the entire domain. Furthermore a more dense mesh is used in the vicinity of the body force distribution. For the highest frequency case $(F=35)$ the grid density corresponds to approximately 20 cells per TS wavelength. Finally, a buffer region of smoothly increasing cell size in streamwise direction is added at the downstream end of the computational domain in order to avoid reflections from the outflowing disturbances.
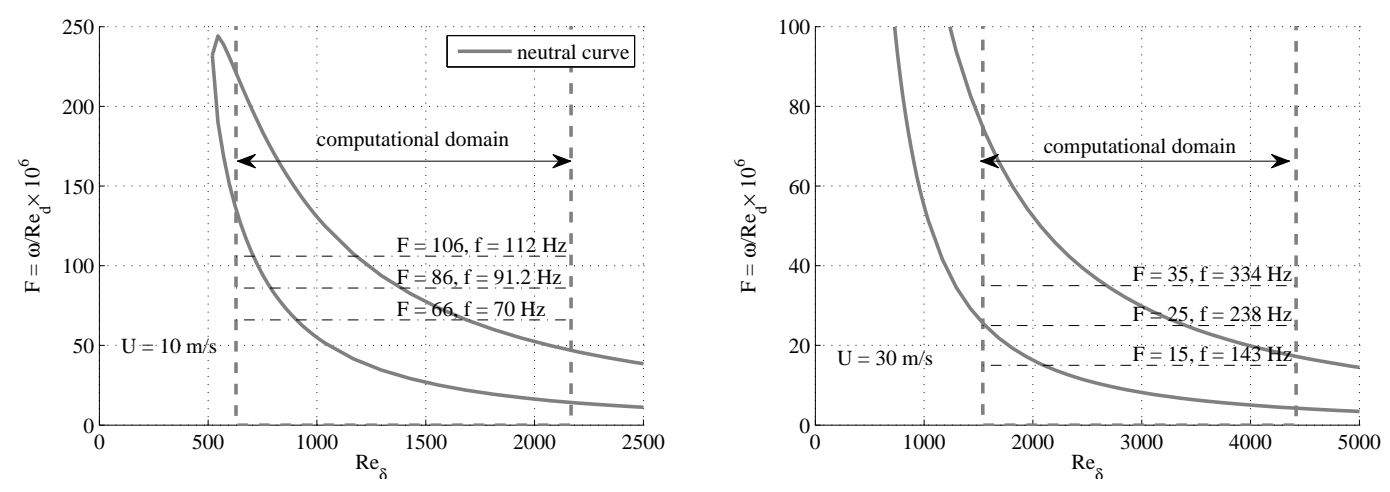

Figure 4. Stability diagrams for cases $A$ and $B$ with respective modes.

The controller settings are mainly fixed for the two cases apart from the convergence constant $\beta$ which is adjusted per sub-case in order to achieve the best control performance. For case A the reference sensor is located at $x=0.4 \mathrm{~m}$ while the error sensor is at $x=0.7 \mathrm{~m}$. The actuator (body force distribution) is located at $x=0.5 \mathrm{~m}$. For case B the reference sensor is located at $x=0.7 \mathrm{~m}$ while the error sensor is at $x=$ $1 \mathrm{~m}$. The actuator (body force distribution) is located at $x=0.8 \mathrm{~m}$. For both cases the FIRI and FIRII filters employ 40 weight coefficients and the sampling rate of the controller is $1 \mathrm{kHz}$.

\section{Results}

\section{A. Verification and modeling considerations}

The numerical framework in this study is comprised of three discreet computational modules which are coupled together in order to achieve the final transition control goal. These are the flow solver, the forcing mechanism and the adaptive control system. Due to the multiple models used in this approach, it is desirable to identify, evaluate and address possible sources of error. This is done via a verification and validation approach wherever is possible.

The flow solver is the most straightforward component to verify and validate since accurate solutions exist for both the mean and the fluctuating velocity components in the form of the Blasius equations and Linear Stability Theory (LST). It should be noted here that both the Blasius solution for the boundary layer and LST are solutions of a different set of equations than the ones the solver of this study handles. Modeling errors are therefore expected.

For verification purposes two grid studies are employed. Firstly a mesh refinement study is conducted. Three computational meshes of different sizes $(106 \times 544,141 \times 727,211 \times 1090$ cells respectively) are created employing the same physical dimensions and cell clustering ratio. The $h$ factor between the three meshes is 1, 1.5 and 2 respectively. To be noted that the cell spacing of mesh 2 is the one used in this study for the investigation of the selected test cases. For all three meshes, the complete control sequence of cases is run. More specifically, for each mesh a mean flow is calculated, the stability case is run for a single frequency case and finally a control case is run employing the generic body force distribution. Several quantities are calculated in order to estimate the observed order of accuracy as defined by Roy. ${ }^{22}$ Fig. 5 presents $N$ factors for the three different meshes for the case of $F=86$. The observed order of accuracy for the $N$ factor at $x=0.8$ is 2.03. Additionally a comparison between the shape of the propagating TS wave is given in the same figure. 
The second grid study involves the location of the top boundary of the computational domain and its influence on the stability simulation. Fig.6 presents the computed $\mathrm{N}$ factors for four cases of top boundary located at different distances from the flat plate wall. It is apparent that a threshold of approximately $25 \delta^{*}$ exists above which the top boundary does not influence the simulation. To be noted here that the top boundary study is done using the spacing of mesh 1 , hence the large difference of the $N$ factor from LST results.
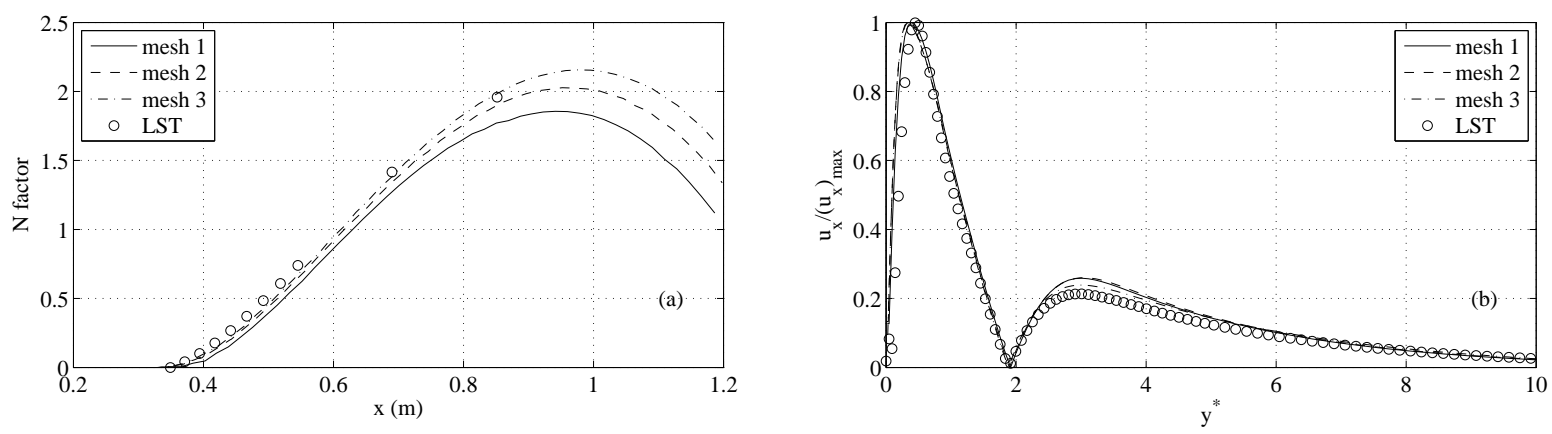

Figure 5. (a) $N$ factor for the three tested grids $(F=86)$ along with LST prediction and (b) shape of the $u$ disturbance for the three meshes along with LST solution.

In contrast to the flow solver, body force model presents difficulties in verification and validation. Nevertheless, several remarks can be made on the possible modeling errors. The representation of the plasma actuator through a volume distributed body force has been a popular method for implementing this type of devices in numerical studies. Yet it should be stressed here that such models must be used with caution especially for high receptivity areas such as the transition domain. It has been shown numerically ${ }^{23}$ and experimentally ${ }^{24}$ that during continuous and pulse operation the plasma actuator induces fluctuating velocity components corre-

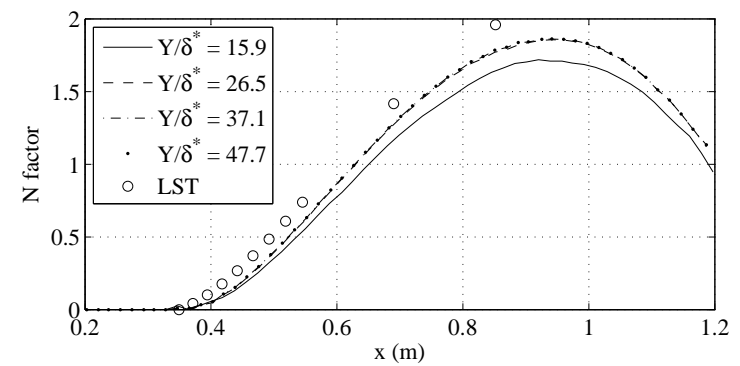

Figure 6. $N$ factor for different top boundary location. $(F=86, \operatorname{mesh} 1)$ sponding to the carrier frequency used for operation.

Most body force models neglect these fluctuations since the usually high carrier frequency ensures that they are decoupled from the time scale of the flow to be controlled. The experimental body force distributions used in this study are based on exactly such an assumption. This presents an upper threshold of Reynolds numbers in which this force model can be used. For example the stability case with $F=35$ corresponds to a natural frequency of $340 \mathrm{~Hz}$ in the case of $U_{\infty}=30 \mathrm{~m} / \mathrm{s}$. This is the highest frequency the authors considered safe to be used here since the carrier frequency of the actuator is set at $2 \mathrm{kHz}$. For higher instability frequencies which are comparable to the carrier frequency of the actuator, a model that takes the latter into account is necessary in order to accurately capture the effect of the actuator.

\section{B. Parametric study on control performance}

Prior to the actual control cases, a parametric investigation on the operation of the controller is performed. Two separate studies are conducted involving the convergence constant $(\beta)$ and the streamwise length of the generic body force distribution $l$. For both cases all other parameters are kept constant.

For the investigation of the convergence constant the single frequency case $F=86$ is used. The generic force distribution employs $l=0.02 \mathrm{~m}$ and $y=0.003 \mathrm{~m}$. The error signal for all tested values of $\beta$ is shown in Fig.7 along with the respective amplitude of the body force as selected by the controller. All cases have a system identification procedure for the first $0.2 \mathrm{~s}$ of simulation based on identical settings. The results are shown here only for the control period (starting at $t=0.2 \mathrm{~s}$ ). Two effects are evident here which are typical for the FXLMS algorithm. Firstly, if the convergence coefficient is too low the algorithm is converging to the optimum solution slowly and in some extreme cases $(\beta=0.0025)$ it might stop converging all together. 
On the other hand if the convergence constant is too large then the algorithm becomes unstable and the force amplitude starts to oscillate around the near optimal value $(\beta=0.02)$. Although the controller still manages to reduce the error signal, the performance is degrading. In a more extreme case $(\beta=0.04)$ the controller fails altogether with very large force values which respectively increase the error.

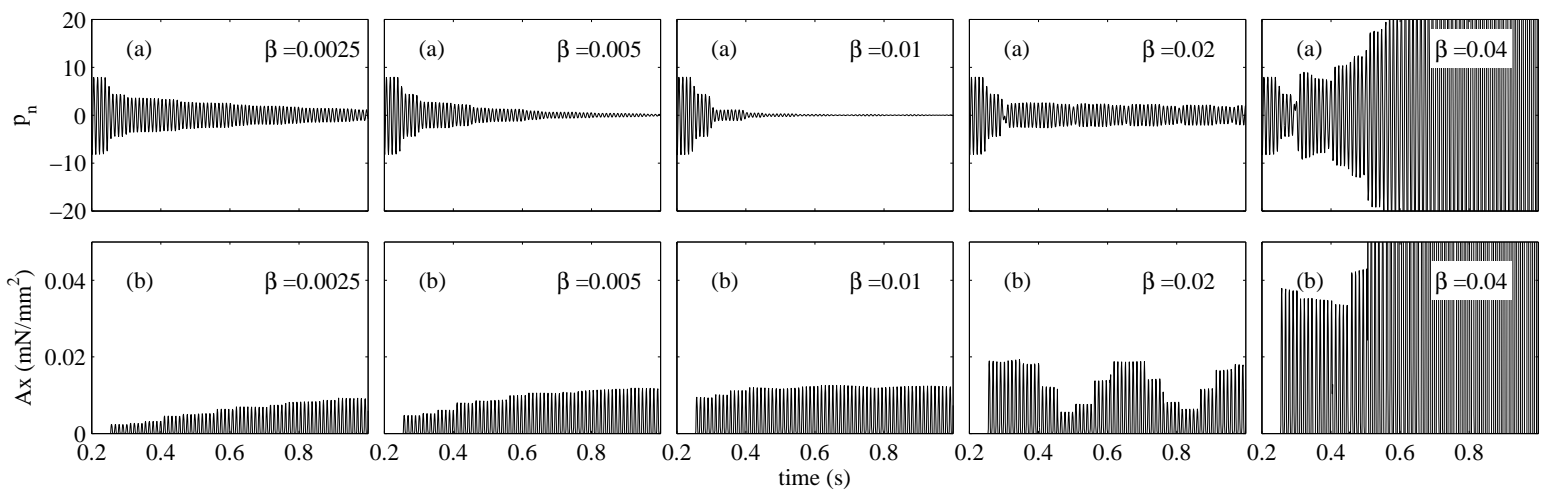

Figure 7. (a) Signal measured by the error sensor and (b) respective body force actuation value for different convergence constant $(\beta) .(F=86)$.

The second parameter investigated is the streamwise length of the force distribution. This is an important value in the transition control efficiency as the length of the optimum forcing distribution is expected to be strongly dependent on the wavelength of the incoming TS wave. Two more factors increase the complexity of this interaction further. Firstly, in cases of natural transition control, more than one frequencies of unstable modes are expected in the boundary layer. As such the wavelength of the modes in the wavetrain is not constant. Secondly, in the case of plasma actuation, the length of the forcing region is coupled to the applied voltage and the amplitude of the forcing itself as can be seen in Fig.3. Combining these two effects, the force distribution in normal operation conditions will have almost certainly sub-optimal characteristics. More discussion on this topic is given in section D. Results for all tested values of $l$ and $l_{n}$ is shown in Fig.8. Here $l_{n}$ is the force length $l$ normalized with the streamwise wavelength of the incoming TS wave $(0.038 \mathrm{~m}$ for $F=86$ ). For all cases $\beta$ is 0.01 .
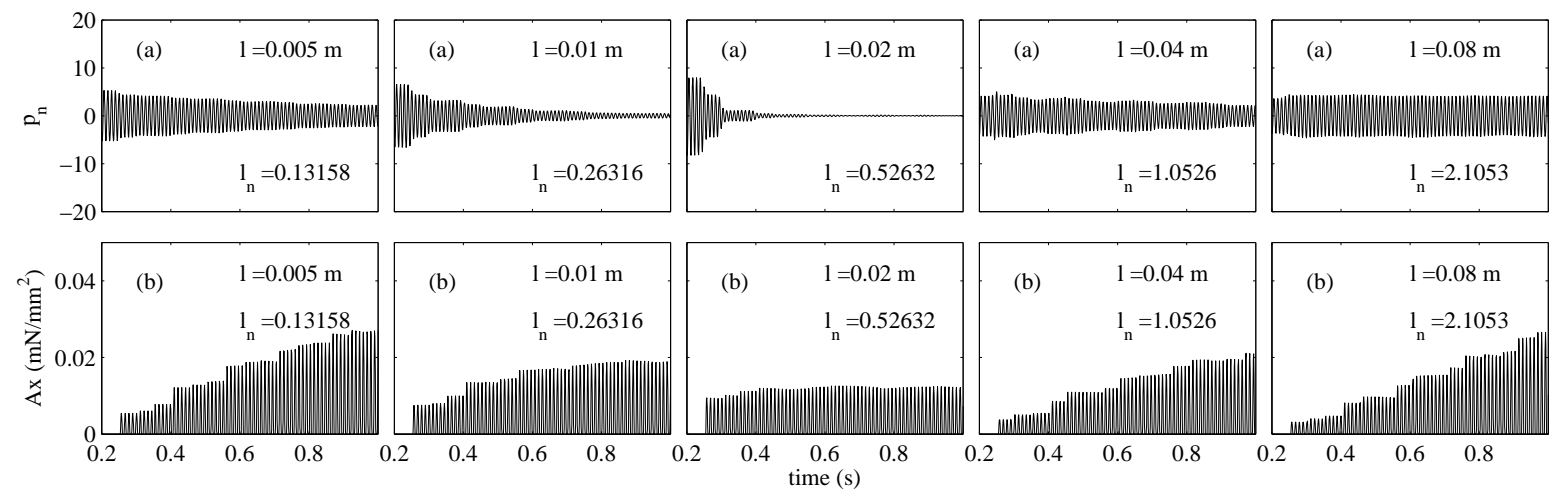

Figure 8. (a) Signal measured by the error sensor and (b) respective body force actuation value for different body force lengths $(l)$. $(F=86)$.

The results indicate that force lengths around half the incoming TS wavelength produce the best control performance. This is partly expected as the TS wave is fluctuating in $x$ with half the wavelength occupied by positive velocity and the other half by negative velocity. Since the force is always positive and directed downstream, destructive interference can only occur when the negative velocity half-wavelength is over the actuator. For larger force lengths $(l=0.04$ and $0.08 \mathrm{~m})$ the controller fails to reduce the error while for smaller lengths $(l=0.005$ and $0.01 \mathrm{~m})$ error reduction is registered although the performance is suboptimal. 


\section{Control case A: Generic force distribution}

The generic force distribution is applied in case A. Three control scenarios are investigated comprising of two single frequency and one multi frequency sub-cases. The controller is operating in single frequency cases corresponding to $F=66$ and 86 while the multi frequency case consists of a wavetrain which is a combination of modes $F=66,86$ and 106 .

Snapshots of the velocity disturbance flowfield are presented in Figs.9 and 10 for the cases of $F=66$ and $F=86$ respectively. Additionally the value of the maximum horizontal velocity component are presented in Fig.11. The comparison between the controlled and uncontrolled cases indicates the effect of the body force actuation. Both the horizontal and vertical components of the disturbance velocity are reduced downstream the actuation region. In the case of $F=66$ amplification of the residual disturbances continues after the actuation since the entire domain is located within the instability region. On the contrary the case of $F$ $=86$ appears to have an almost total wave cancellation since the TS waves cross the upper neutral curve approximately after $x=0.9$. To be noted here that the settings of the controller are kept the same for these two cases apart from the convergence constant $\beta$. This is adjusted specifically for each case in order to achieve optimal performance from the controller.

Apart from the reduction of the fluctuating velocity components, an interesting aspect is the mean flow component present close to the forcing region. This is an artifact of the unidirectionality of the body force. More specifically, the generic body force is restricted to take only positive (downstream) values in the case where the controller instructs it to be negative. This is to ensure that the overall forcing mechanism resembles the unidirectional plasma actuation. In both single frequency cases the mean flow disturbances appears to convect and diffuse downstream.
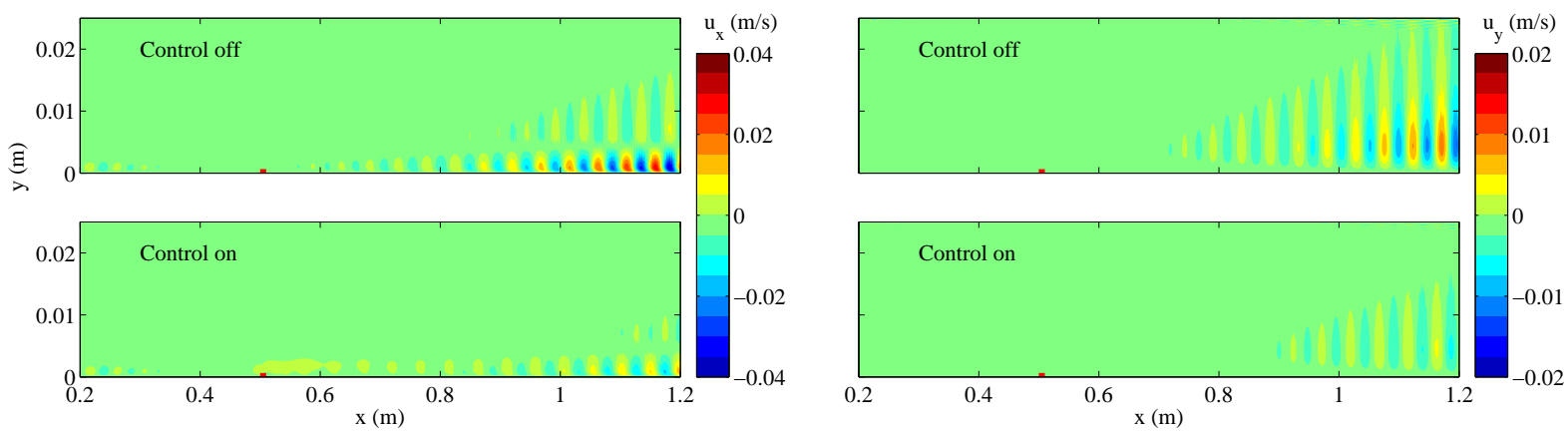

Figure 9. Snapshots of horizontal $\left(u_{x}\right)$ and vertical $\left(u_{y}\right)$ components of disturbance velocity with and without control. $(F=66)$. (Position of the actuator is indicated by the small red rectangle)
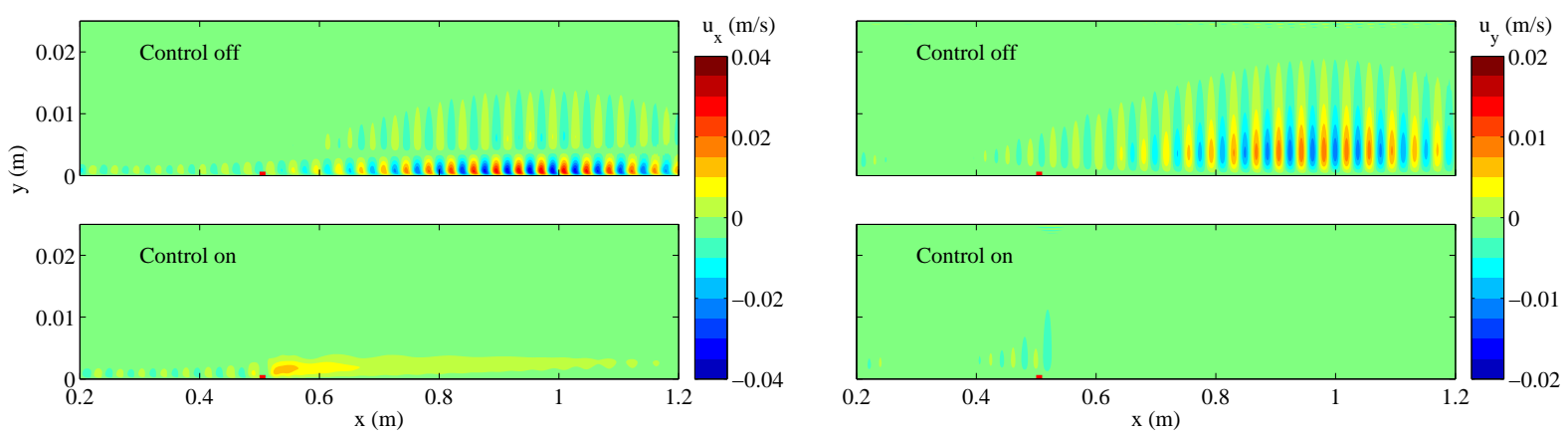

Figure 10. Snapshots of horizontal $\left(u_{x}\right)$ and vertical $\left(u_{y}\right)$ components of disturbance velocity with and without control. $(F=86)$.(Position of the actuator is indicated by the small red rectangle)

Velocity field snapshots for the multi frequency sub-case are presented in Fig.12. Additionally the maximum horizontal velocity component is presented in Fig.11. This case involves the development of a wavetrain consisting of three modes at $F=66,86$ and 106 respectively. Contrary to the single frequency cases the disturbances are introduced in the domain via a distributed body force as previously explained. The weights 
for all the modes are set to be equal.
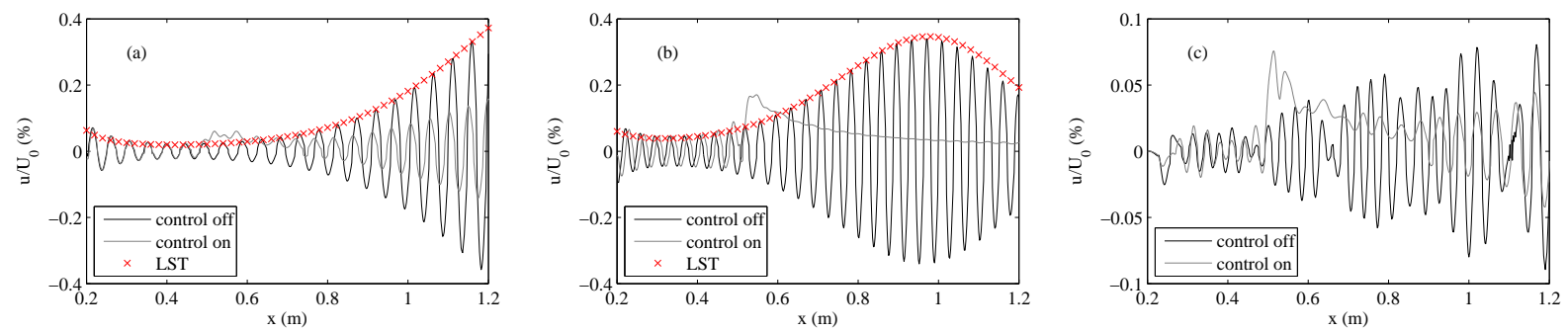

Figure 11. Normalized maximum horizontal velocity of disturbance with and without control for the (a) case $F=66,($ b) case $F=86$ and (c) multi frequency case $F=66,86,106$.

It is apparent here that while the controller still manages to reduce the amplitude of the traveling wavetrain, total wave cancellation is not possible. This can be explained by considering the nature of the forcing mechanism. Although the controller attempts to regulate the amplitude of the imposed body force, no authority exists over the spatial distribution, location and shape of the body force. On the other hand the incoming disturbances present multiple wavelengths, amplitudes and frequencies. As was shown in section $\mathrm{B}$ the wavelength of the imposed force has considerable influence on the final performance of the controller. It is thus expected that in a scenario where multiple frequencies are to be controlled a predefined force distribution will lead the controller to a sub-optimal operation stage. This is of high importance in actual plasma cases where the spatial extent and shape of the force distribution cannot be explicitly controlled as it is a function of the applied voltage. This is further demonstrated in case B.
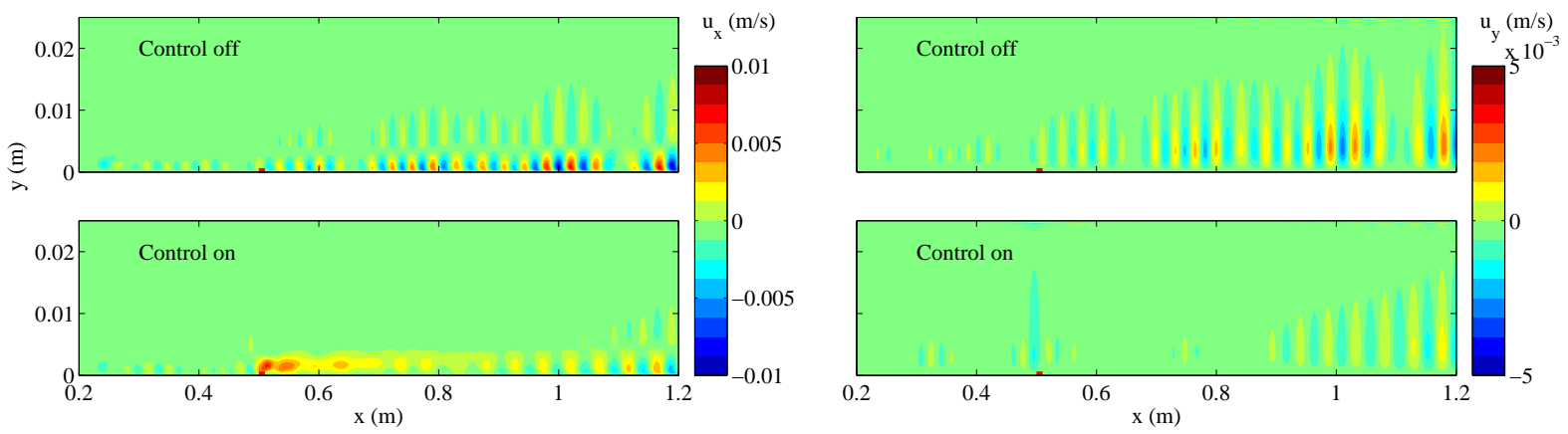

Figure 12. Snapshots of horizontal $\left(u_{x}\right)$ and vertical $\left(u_{y}\right)$ components of disturbance velocity with and without control for the multi frequency sub-case $(F=66,86,106)$.(Position of the actuator is indicated by the small red rectangle)

The temporal evolution of the reference and error signal along with the respective amplitude of the body force $\left(A_{x}\right)$ are shown in Figs.13-15 for the two single frequency cases and one multi frequency case respectively. As already mentioned the system identification time is $0.2 s$ in all cases. During this time the body force is directly driven by the reference pressure signal while the system identification algorithm builds the digital representation of the cancellation path. It is interesting to note that during this stage the error signal is reduced in the case of $F=66$ while it is increased in the case of $F=86$. This emphasizes the role of the system ID part in which a random signal which can have either positive or negative effects can be used to drive the forcing mechanism.

The difference in control performance between the two single frequency cases is also evident here. Apart from the final reduction in amplitude, the convergence of the controller to the final operation state is considerably faster for the case of $F=86$. This again has to do with the relation between the spatial extent and shape of the body force distribution compared to the wavelength of the incoming instability.

For the multi frequency case results indicate that although the controller converges relatively fast to the final state of operation, the complete elimination of the fluctuating disturbances is not possible. 

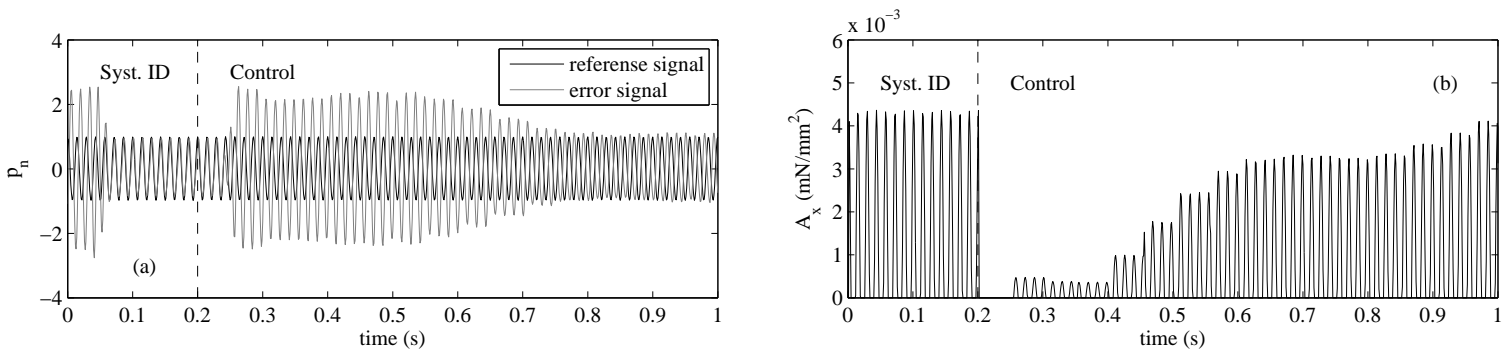

Figure 13. (a) Normalized reference and error signals during controller operation and (b) respective body force amplitude $\left(A_{x}\right)$ for the case of $F=66$
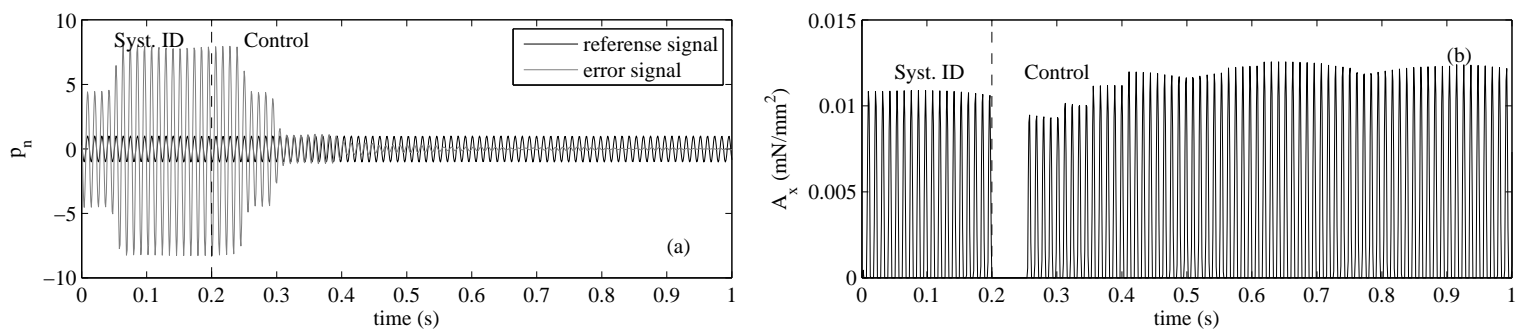

Figure 14. (a) Normalized reference and error signals during controller operation and (b) respective body force amplitude $\left(A_{x}\right)$ for the case of $F=86$
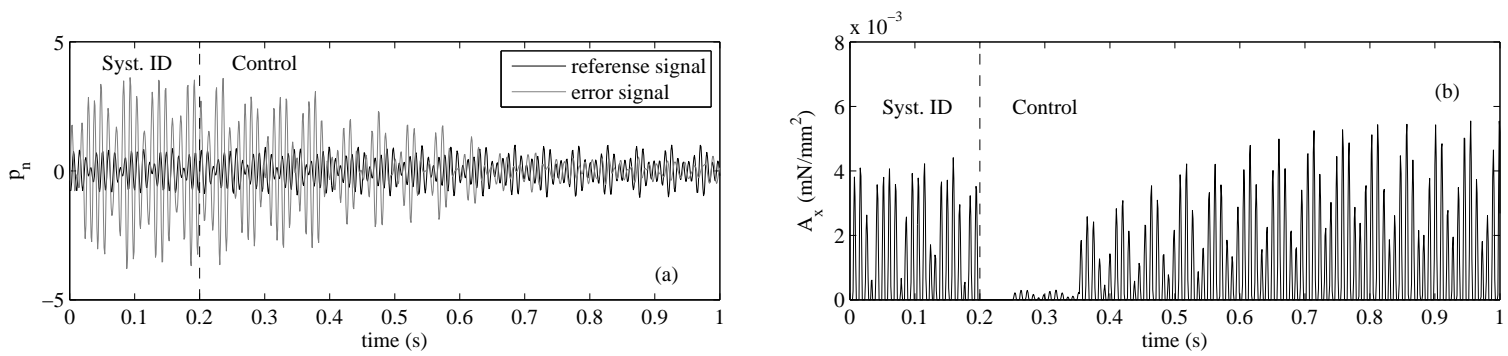

Figure 15. (a) Normalized reference and error signals during controller operation and (b) respective body force amplitude $\left(A_{x}\right)$ for the multi frequency case $(F=66,86,106)$ 


\section{Control case B: Plasma force distribution}

The experimentally derived plasma body force distributions have been implemented in the numerical framework with the use of a continuous voltage function based on Bernstein surface approximations. Due to the strength of the actuator itself, the tested cases involve only the case B conditions where the freestream velocity is $30 \mathrm{~m} / \mathrm{s}$. Additionally the actuator is placed more downstream in order to demonstrate the ability to tackle heavily amplified disturbances.

Two sub-cases are studied, namely a single frequency case with mode $F=25$ and a multi frequency case with modes $F=15,25,35$ of equal strength at the disturbance introduction. Snapshots of the evolved flowfield for the single frequency case are shown in Fig.16 while the respective maximum amplitude of the disturbance is shown in Fig.18. Some comments can be made on this case. The stability characteristics of this case are significantly more adverse than the sub cases of case A. Mode $F=25$ has an $\mathrm{N}$ factor which almost reaches 9 by the end of the computational domain. This signifies the transitional state of this boundary layer. Additionally, at approximately $x=1.1$ the first harmonic of the mode starts to show significant values departing the maximum disturbance amplitude from the sinusoidal shape.

The controller achieves significant reduction on the amplitude of the TS mode. Nevertheless, downstream of the actuator the boundary layer is already deep in the instability region and the residual disturbances after actuation quickly amplify again. This effect raises the importance of multiple successive actuators in the case of practical applications.

Snapshots of the flowfield for the multi frequency case are presented in Fig17 while the maximum amplitude of the disturbance is shown in Fig.18. It is evident that the actuator can respond well to the existence of multiple frequencies in the spectrum of TS waves. Additionally a pronounced mean flow disturbance is evident which nevertheless is significantly reduced within approximately 6 TS wavelengths downstream.
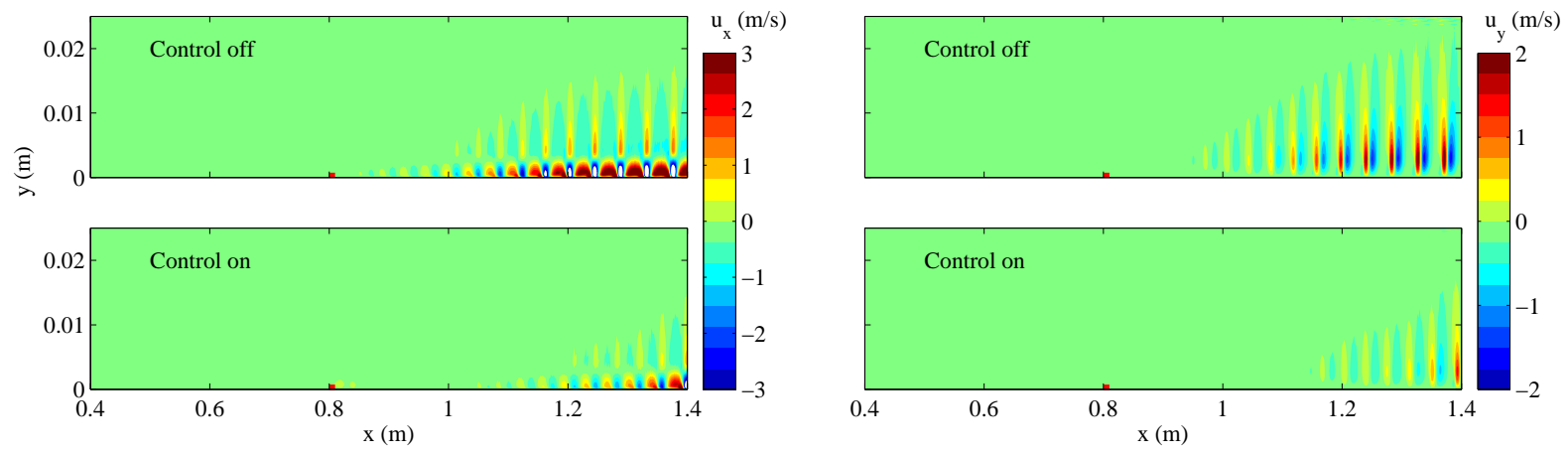

Figure 16. Snapshots of horizontal $\left(u_{x}\right)$ and vertical $\left(u_{y}\right)$ components of disturbance velocity with and without control. $(F=25)$. (Position of the actuator is indicated by the small red rectangle)
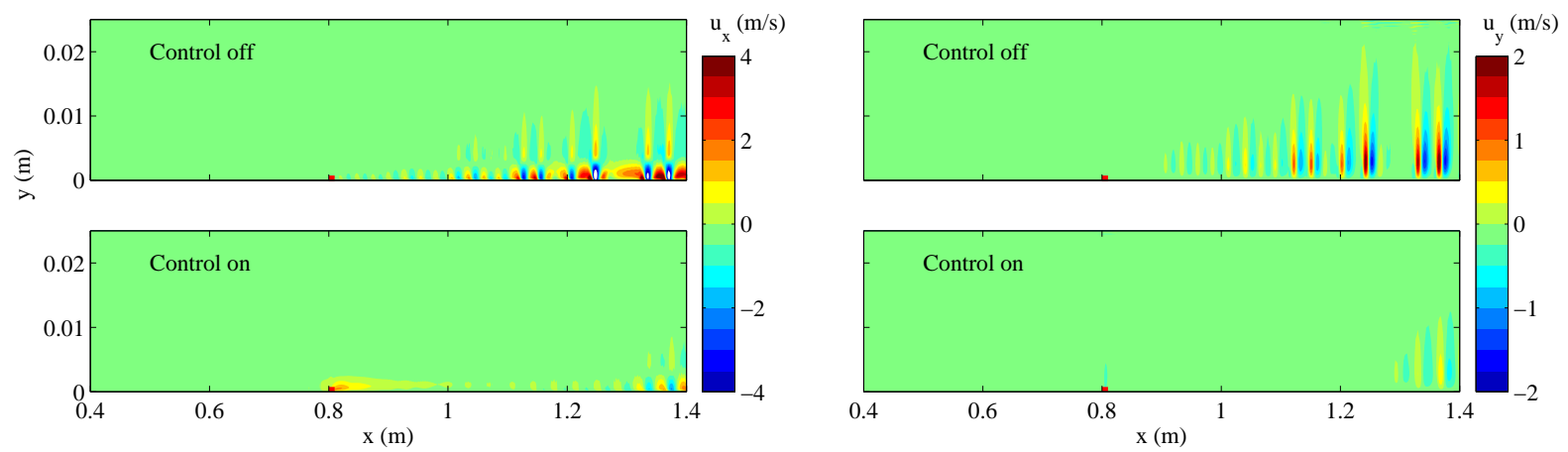

Figure 17. Snapshots of horizontal $\left(u_{x}\right)$ and vertical $\left(u_{y}\right)$ components of disturbance velocity with and without control for the multi frequency case. $(F=15,25,35)$.(Position of the actuator is indicated by the small red rectangle)

The reference, error and voltage signals as measured by the controller are presented in Figs.19 and 20 

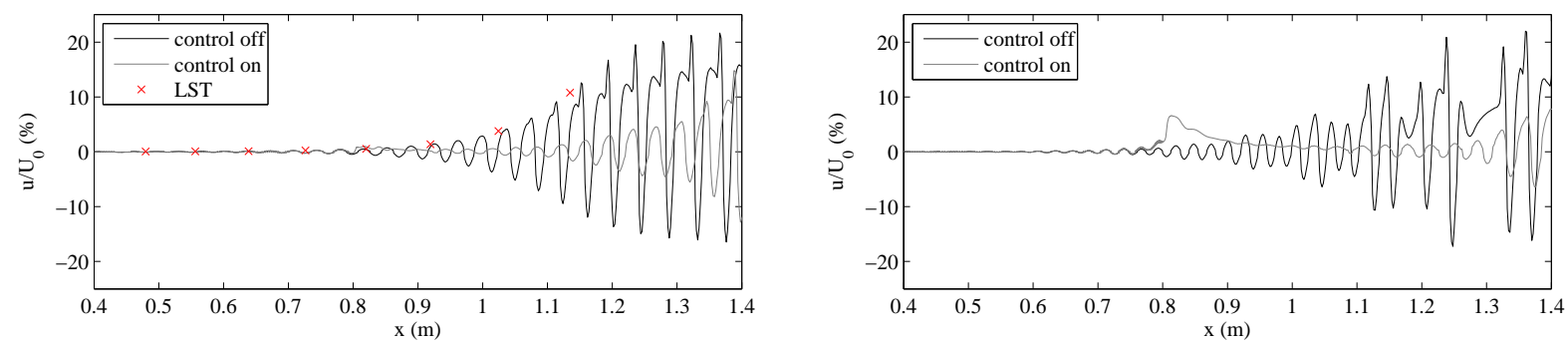

Figure 18. Normalized maximum horizontal velocity of disturbance with and without control for (a) case $F=25$ and (b) multi frequency case $F=15,25,35$

for the single and multi frequency cases respectively. For the single frequency case a relatively large time span is spent in which the actuator does not operate. This is due to the fact the required voltage decided by the control algorithm lies below the threshold value of $8 k V_{p p}$. In these cases the controller automatically restricts the voltage to a zero value.

For the multi frequency case the effect is opposite. Due to the strength of the incoming disturbances the actuator is requested to operate with at voltages that lye above the maximum threshold of $16 \mathrm{k} V_{p p}$. In this case the maximum voltage is restricted to $16 k V_{p p}$. This cutoff voltage effectively renders the actuation signal shape to trapezoidal rather than sinusoidal. In spite this the actuator achieves significant reduction of the TS amplitude.

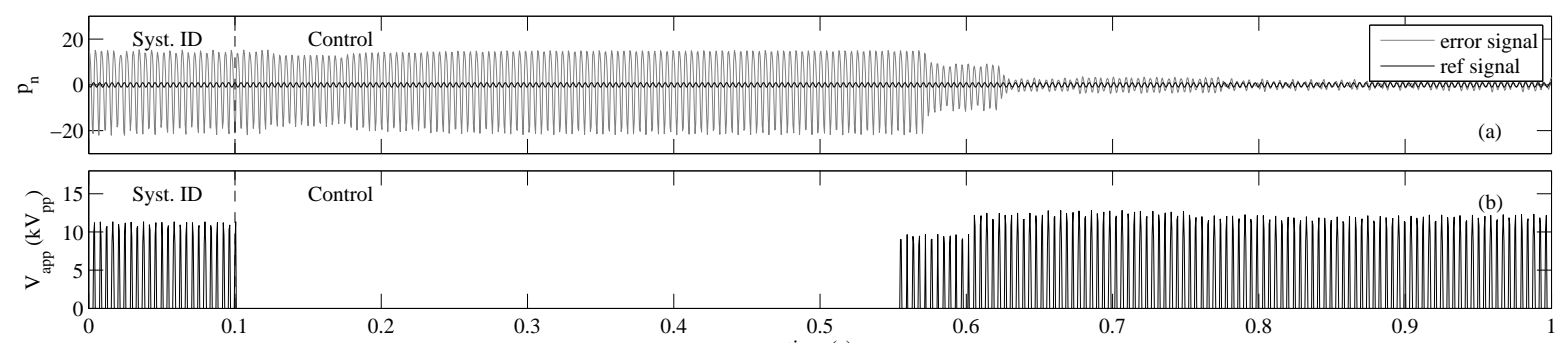

Figure 19. (a) Normalized reference and error signals during controller operation and (b) respective applied voltage value $\left(V_{a p p}\right)$ for the case of $F=25$

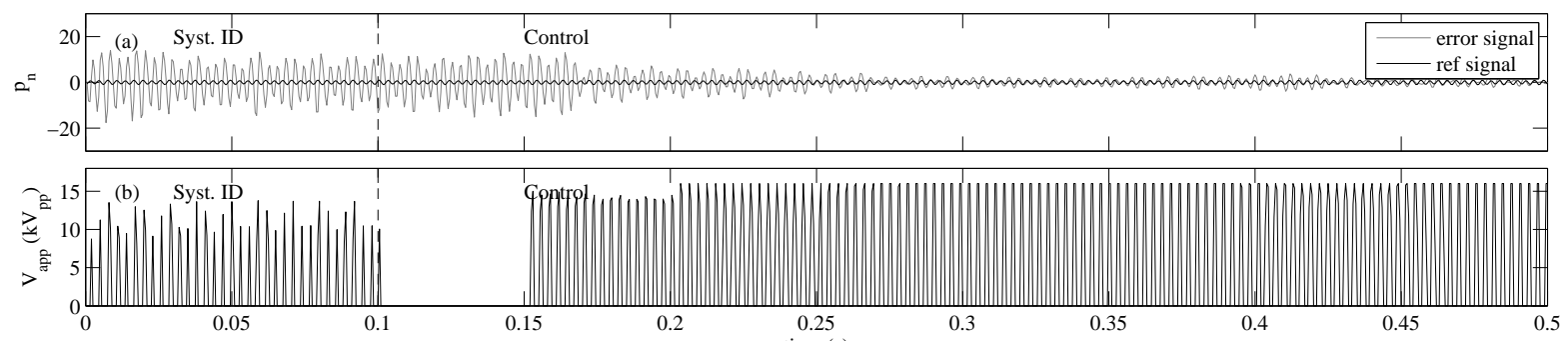

Figure 20. (a) Normalized reference and error signals during controller operation and (b) respective applied voltage value $\left(V_{a p p}\right)$ for the case of $F=15,25,35$

An overview of the test cases, respective force model and TS strength reduction is presented in Tab. Here $\Delta u_{\max }$ is the reduction of the maximum horizontal velocity component of the TS mode at $x=1 \mathrm{~m}$ while $\Delta p_{e}$ is the reduction of the error signal as this is registered by the controller.

\section{Conclusions}

A numerical investigation on the use of plasma actuators for transition control is presented. The numerical framework involves the solution of the full unsteady 2D incompressible Navier Stokes equations using a finite 
Table 1. Overview of test cases with respective force model and reduction in TS wave strength.

\begin{tabular}{l|r|r|r|r}
\hline \hline case & frequency $(F)$ & force & $\Delta u_{\max }$ at $x=1 \mathrm{~m}(\%)$ & $\Delta p_{e}(\%)$ \\
\hline A. 1 & single $(66)$ & generic & 61 & 55 \\
A. 2 & single $(86)$ & generic & 90 & 98 \\
A. 3 & multi $(66,86,106)$ & generic & 62 & 69 \\
B. 1 & single $(25)$ & plasma & 84 & 79 \\
B. 2 & multi $(15,25,35)$ & plasma & 83 & 93 \\
\hline \hline
\end{tabular}

volume formulation. Furthermore, an adaptive control system based on the filtered-x LMS algorithm is implemented directly into the flow solver. The control system uses pressure signal at the wall in order to compute the frequency, phase and amplitude of the plasma body force which minimizes the intensity of the propagating TS waves. Additionally, the effect of plasma actuators has been modeled using volume distributed body forces. A generic model as well as experimentally measured force distributions have been used. Results show large reductions in wave amplitude.

\section{References}

${ }^{1}$ Post, M. L. and Corke, T. C., "Separation control on high angle of attack airfoil using plasma actuators," AIAA Journal, Vol. 42, No. 11, 2004, pp. 2177-2184.

${ }^{2}$ Jukes, T. N., Choi, K. ., Johnson, G. A., and Scott, S. J., "Turbulent drag reduction by surface plasma through spanwise flow oscillation," 3rd AIAA Flow Control Conference, Vol. 3, 2006, pp. 1687-1700.

${ }^{3}$ Jacob, J. D., Rivir, R., Carter, C., and Estevadeordal, J., "Boundary layer flow control using AC discharge plasma actuators," 2nd AIAA Flow Control Conference, 2004.

${ }^{4}$ Huang, J., Corke, T. C., and Thomas, F. O., "Plasma Actuators for Separation Control of Low-Pressure Turbine Blades," AIAA Journal, Vol. 44, 2006, pp. 51-57.

${ }^{5}$ Seraudie, A., Aubert, E., Naude, N., and Cambronne, J. P., "Effect of plasma actuators on a flat plate laminar boundary layer in subsonic conditions," 3rd AIAA Flow Control Conference, Vol. 2, 2006, pp. 1065-1073.

${ }^{6}$ Grundmann, S. and Tropea, C., "Active cancellation of artificially introduced Tollmien-Schlichting waves using plasma actuators," Experiments in Fluids, Vol. 44, No. 5, 2008, pp. 795-806.

${ }^{7}$ Moreau, E., "Airflow control by non-thermal plasma actuators," Journal of Physics D: Applied Physics, Vol. 40, No. 3, 2007, pp. 605-636.

${ }^{8}$ Corke, T. C., Post, M. L., and Orlov, D. M., "SDBD plasma enhanced aerodynamics: concepts, optimization and applications," Progress in Aerospace Sciences, Vol. 43, No. 7-8, 2007, pp. 193-217.

${ }^{9}$ Jayaraman, B., Cho, Y. ., and Shyy, W., "Modeling of dielectric barrier discharge plasma actuator," 38th AIAA Plasmadynamics and Lasers Conference, Vol. 2, 2007, pp. 1019-1038.

${ }^{10}$ Lagmich, Y., Callegari, T., Pitchford, L. C., and Boeuf, J. P., "Model description of surface dielectric barrier discharges for flow control," Journal of Physics D: Applied Physics, Vol. 41, No. 9, 2008.

${ }^{11}$ Jayaraman, B. and Shyy, W., "Modeling of dielectric barrier discharge-induced fluid dynamics and heat transfer," Progress in Aerospace Sciences, Vol. 44, No. 3, 2008, pp. 139-191.

${ }^{12}$ Singh, K. P., Roy, S., and Gaitonde, D. V., "Modeling of dielectric barrier discharge plasma actuator with atmospheric air chemistry," Vol. 2, 2006, pp. 576-586.

${ }^{13} \mathrm{He}, \mathrm{C} .$, Corke, T. C., and Patel, M. P., "Numerical and experimental analysis of plasma flow control over a hump model," Tech. rep., 2007.

${ }^{14}$ Kotsonis, M., Ghaemi, S., Veldhuis, L., and Scarano, F., "Measurement of the body force field of plasma actuators," Journal of Physics D: Applied Physics, Vol. 44, No. 4, 2011, Cited By (since 1996): 1.

${ }^{15}$ Schlichting, H. and Gersten, K., Boundary Layer Theory, Springer, 2000.

${ }^{16}$ Sturzebecher, D. and Nitsche, W., "Active cancellation of Tollmien-Schlichting instabilities on a wing using multi-channel sensor actuator systems," International Journal of Heat and Fluid Flow, Vol. 24, No. 4, 2003, pp. 572-583.

${ }^{17}$ Thomas, A., "Control of boundary-layer transition using a wave-superposition principle." Journal of Fluid Mechanics, Vol. 137, 1983, pp. 233-250.

${ }_{18}$ Albrecht, T., Grundmann, R., Mutschke, G., and Gerbeth, G., "On the stability of the boundary layer subject to a wall-parallel Lorentz force," Physics of Fluids, Vol. 18, No. 9, 2006, Cited By (since 1996): 2.

${ }^{19}$ Bower, W. W., Kegelman, J. T., Pal, A., and Meyer, G. H., "A numerical study of two-dimensional instability-wave control based on the Orr-Sommerfeld equation," Physics of Fluids, Vol. 30, No. 4, 1987, pp. 998-1004.

${ }^{20}$ Kuo, S. and Moran, D., Active noise control systems, John Wiley, New York, 1996.

${ }^{21}$ Hansen, C., Understanding Active Noise Cancellation, Tylor and Francis, 2001.

${ }^{22}$ Roy, C. J., "Review of code and solution verification procedures for computational simulation," Journal of Computational Physics, Vol. 205, No. 1, 2005, pp. 131-156, Cited By (since 1996): 77. 
${ }^{23}$ Likhanskii, A. V., Shneider, M. N., MacHeret, S. O., and Miles, R. B., "Modeling of dielectric barrier discharge plasma actuator in air," Journal of Applied Physics, Vol. 103, No. 5, 2008.

${ }^{24}$ Benard, N. and Moreau, E., "Capabilities of the dielectric barrier discharge plasma actuator for multi-frequency excitations," Journal of Physics D: Applied Physics, Vol. 43, No. 14, 2010. 